\title{
The Power and Perspective of Mommy Bloggers: Formative Research with Social Media Opinion Leaders about HPV Vaccination
}

\author{
Amelia Burke-Garcia \\ George Mason University \\ aburkega@gmu.edu
}

\author{
Corinne N. Berry \\ George Mason University \\ coriberr@gmail.com
}

\author{
Gary Kreps \\ George Mason University \\ gkreps@gmu.edu
}

\author{
Kevin Wright \\ George Mason University \\ kwrigh16@gmu.edu
}

\begin{abstract}
Since the development of the Human Papillomavirus (HPV) vaccine in 2006, significant promotion efforts have targeted adolescent females and their parents in order to reduce the incidence of $H P V$ and related cancers. Yet vaccination rates for pre-adolescents (the recommended age of administration) still lag behind. Social media and social network campaigns hold promise not only for promoting broad awareness but also for influencing vaccination attitudes and behaviors by utilizing opinion leaders for message dissemination. This formative intervention study explored the feasibility of leveraging online 'mommy bloggers' as thought leaders and sources of influence in the promotion of $H P V$ vaccine messages. Findings include insights into vaccine hesitancy amongst mommy bloggers; approaches for discussing HPV vaccination in the blogosphere; and engagement strategies for working with mommy bloggers.
\end{abstract}

\section{Introduction}

Human Papillomavirus (HPV) is considered the most common sexually transmitted infection (STI) among men and women in the United States [1]. Approximately 14 million new infections occur each year and the highest rate of new HPV infections is among people 15 to 24 years of age [2]. There are many types of HPVs including low risk forms that can cause genital warts or high-risk types that may lead to cancer [3]. It is estimated that HPV is likely "to be responsible for more than $90 \%$ of anal and cervical cancers, about $70 \%$ of vaginal and vulvar cancers, and more than $60 \%$ of penile cancers" [4].

In June 2006, the Food and Drug Administration approved the world's first HPV vaccine, known as Gardasil $^{\mathrm{TM}}$. Manufactured by Merck \& Co., (Merck) the vaccine received a provisional recommendation from the Advisory Committee on Immunization
Practices (ACIP) for use in females aged 9-26 [5]. To be fully immunized a person must receive a three-dose series, over the course of six months, and it is considered most efficacious when administered prior to the onset of sexual activity [1]. In 2009, the vaccine was approved for "permissive use" in males and guidance revised in 2011 to recommend routine administration to males [6]. The current Centers for Disease Control and Prevention (CDC) recommendation is to begin the series, for both males and females, at the age of 11 or 12 [1].

This paper reviews a pilot intervention study that aimed at exploring the feasibility of leveraging online 'mommy bloggers' as thought leaders and sources of influence in the promotion of HPV vaccine messages. It was developed in the context of a semester-long course at George Mason University. This paper reviews the foundational literature supporting this study, the study design and methods, the findings and future research opportunities. Finally, it acknowledges the limitations of this study.

\section{Literature review}

This section provides the foundation for the study by first reviewing the literature on HPV vaccine acceptance, then looking at how promotion of the HPV vaccine has been conducted, drilling down into the use of social media to support HPV vaccine promotion including the role of mommy bloggers as information sources. It concludes by describing Social Network Theory as the theoretical underpinning of the pilot.

\subsection{HPV vaccine acceptance}

Research suggests that parents prefer to make the vaccination decision in concert with their child and initiate vaccination when it is developmentally appropriate to have the conversation; yet the timing for when the vaccination is recommended in preadolescence presents a challenge [7]. Empirical 
research has also shown that cost, provider recommendation, and general knowledge about HPV, the vaccine and cervical cancer may be predictors of parental attitudes toward vaccination [8]. Parents' decisions about whether to vaccinate for an STI have also been discussed in the context of fear, e.g. parents attempt to balance the tension between protecting their child from a preventable infection and the fear that the vaccination may encourage risky sexual behavior [9][10]. Other socio-demographic factors, such as having an older daughter [11] or having a family member with cancer [12] have also been associated with parental acceptance of the HPV vaccine.

\subsection{HPV vaccine promotion}

In general, HPV promotion efforts have utilized both gain and loss framing [13] with varying degrees of success and messages that address the known socialcognitive antecedents to parent's vaccination decisions [14][15][7]. Direct-to-consumer campaigns have focused primarily on influencing the mother, given her role in health decision-making and the early age of recommended administration [13][15].

At the end of 2006, Merck launched a national multi-media campaign [5] and spent approximately 98 million dollars on measured media for Gardasil ${ }^{\mathrm{TM}}$ the first year [16]. The campaign sought to increase awareness of the link between HPV and cervical cancer and to promote vaccination in females [17]. This campaign utilized messages to elevate perceptions of risk in terms of HPV-related cancers while simultaneously boosting perceptions of control and efficacy through vaccination [18].

Since empirical studies have also shown provider recommendation to be highly instrumental in parental acceptance of HPV vaccination [14][19], the CDC has focused its campaign efforts on the clinical setting. The CDC has prioritized educating providers about the importance of routine administration of the vaccine for both males and females and how to have HPV vaccine conversations with parents and patients - including how to give an effective recommendation [20]. The CDC campaign, You are the Key, reminds providers that they are the key to reducing the risk and incidence of HPV-related cancers in their patients [20].

This significant investment in promotion has resulted in increased uptake of the vaccine, particularly among females, in the last few years [21][18]. However, according to the 2013 National Immunization Survey, uptake among males remains relatively low with only $34 \%$ of adolescent boys receiving one or more doses of any HPV vaccine [22]. Despite medical guidance to vaccinate early, the percentages are even lower for pre-adolescents and studies indicate parents prefer to delay vaccination for females in particular [8][23][10].

Lower vaccination rates in males have been attributed to the early promotion efforts that framed HPV as a "woman's issue" [24]. Moreover, since promotion efforts have been primarily executed through mass media and healthcare providers, other potential sources of influence have gone unattended. To date, little attention has been paid to the efficacy of using interpersonal channels, such as social and community networks, to influence acceptability and uptake of the HPV vaccine.

\subsection{Social media and HPV}

The dialogic nature of social media allows senders to reach broad audiences and receivers to get involved in the conversation. The few studies that examined HPV vaccine promotion through social media indicate great potential for using blogs, Twitter and other online communication channels, not only for increasing awareness but also to influence decision making [25][26].

For over a decade, consumers have been increasingly seeking 'active channels' like the Internet as a primary source of health information - particularly for the most health conscious [27]. Mothers especially are using the Internet and social media to both inform and discuss health-related decisions for their children [28]. In terms of health information, $86 \%$ of women report that they make the decisions about healthcare treatments for their entire family [29]; and one-third of moms search the Internet for health information once a day or every few days [29]. Bailey further supports the idea that health communicators should go where moms spend time, which increasingly means online social network and blogging sites [30].

\subsection{Mommy blogs and bloggers defined}

There are about 3.9 million moms in the United States who identify as bloggers [31]. A mommy blogger is defined as, "A mother who blogs about her children, motherhood, parenting or related topics" [32]. The average mommy blogger is 37 years old and almost $90 \%$ of mommy bloggers have kids between the ages of 2 and 11 [31].

Mommy bloggers have traditionally been white, middle-to-upper class, educated mothers with the average mommy blog user's household income [ranging from] $\$ 14,000-\$ 84,000$, which is higher than the average income level for non-blogging moms [31]. As well, moms who read or contribute to blogs are also $52 \%$ more likely to have college degrees than moms who do not [31]. This suggests that "mommy 
bloggers belong to a pretty elite social set" [31]. However, increasingly, mommy bloggers are becoming more diverse in ethnicity/race and niche topic areas [33].

\subsection{Mommy blogs as information sources}

With $14 \%$ of all American mothers with at least one child in their household turning to blogs for advice [31], mommy blogs are important sources of information about a variety of health-related topics. According to eMarketer, parenting and pregnancy websites are the top source moms and expectant moms use to learn about products and services [34]. Furthermore, mommy blogs and website may also serve as a source of social support, connection and validation for women transitioning into motherhood and navigating health decisions for their children for the first time [35][36][37].

The availability of vast amounts of health information online has created a factor of convenience as well. Today, mothers' are able to easily seek out vaccination information prior to, between and following visits with their healthcare provider [38]. A recent analysis suggests that parents' social networks play a significant role in vaccine decision-making [39]. This notion is further supported by research that suggests that parents may rely heavily on advice from personal networks in vaccine decision-making [40]. Finally, data from Pew suggests that while clinicians are still the top source of health information in the U.S., online information, curated by peers, is a significant supplement [41].

As a 'one stop shop' for entertainment, creative ideas and useful information (including health), it is no surprise that mommy blogs have become so popular. Some of the most successful mommy bloggers tout thousands of followers/readers and earn their living through blogging [42]. Seen as a trusted member of this virtual mommy community, their readers rely upon them for information, news and opinion often looking to them as 'opinion leaders' [43]. The value of these mommy blogs should not be overlooked by public health communicators and should be further explored as sources of interpersonal influence in mothers' decisions about vaccination.

\subsection{Social network theory}

Social Network Theory (SNT) serves as the ideal theoretical frame for this study, given the focus on opinion leaders in online social networks, specifically the mommy blogosphere. SNT suggests that people access personal communication networks in order to access relevant information and support from others
[44]. Specifically, personal network relationships can help members access social cues, provide people with a sense of belonging, form their identity, and gain a sense of protection from being around others like them [35][44]. This is particularly salient for new and expecting mothers undergoing identity transitions and seeking social support from those more experienced in motherhood [36].

Prior research supports this, showing that eHealth applications have the opportunity to connect consumers through social networking; and that health communication generally has been "person-directed" but the process of initiating and maintaining a life change is made in the context of family, community, and other cultural factors [45]. Therefore, incorporating health communication into a life context may also enable people to make changes across a range of health issues; and this approach is likely to be more effective at strengthening the mediators of change: people's sense of efficacy and control to make actual changes [45]. Finally, social network applications are ideally suited for connecting social networks for personally promoting health because they take advantage of the synergistic contributions of mass and interpersonal media needed to effect change on individual, institutional, and social levels [45].

\section{Research questions}

This formative intervention study seeks to explore the feasibility of utilizing mommy bloggers as opinion leaders in a HPV immunization campaign. To accomplish this, the researchers hope to better understand mommy blogger perspectives on HPV vaccination (pre- and post-intervention), how they interpret and decide to communicate about the issue with their readers, and ultimately the response or dialogue that ensues with their readers. In pursuit of these goals, the following research questions were posited:

RQ1: How are mommy bloggers' knowledge and attitudes about HPV vaccination affected by the intervention?

RQ2: How do mommy bloggers frame HPV vaccine promotion messages to their readership?

RQ3: What message effects occur as a result of the blog post (intervention)?

While this is a highly formative study and intervention, the researchers hope to glean some insights that may serve to expand research in this area and inform a larger campaign. 


\section{Method}

\subsection{Design}

This study utilizes an inductive and purposive approach to exploring how mommy bloggers understand and frame HPV promotion messages and the potential impact of a blog post (intervention) on both the bloggers and their readership [46]. The study and intervention was developed in the context of a University semester-long course and was therefore limited in scope. Given the limited timeframe, the study was designed to evaluate process rather than outcome measures and to better understand the mommy bloggers as opinion leaders. A mixed-method approach was utilized to collect both quantitative and qualitative data elements throughout the campaign.

\subsection{Participants}

The study worked with TheMotherhood mommy blogger network to identify and engage its participants. TheMotherhood network includes approximately 14,000 influencers, who are comprised of a mix of Caucasian/white, Latina and African-American bloggers. As well, approximately $1-2 \%$ of the network is comprised of fathers.

4.2.1. Sample. The sample for this study was purposive and selected from a group of 15 mommy bloggers, identified by TheMotherhood network, who expressed interest in participating in a non-paid academic study. The research team reviewed and vetted each blog and contacted nine bloggers to participate. Out of the nine contacted, five consented and participated in the initial questionnaire, with four $(\mathrm{N}=4)$ completing the intervention, and follow up questionnaire.

4.2.2. Mommy bloggers. The participants were comprised of mommy bloggers with 1 to 4 children, both boys and girls, ranging in age from 6 months to 15 years. The participants also ranged in the number of years since they had become a mother (7 months to 11 years). The participants were primarily Caucasian/White (3 of 4) and one was Asian American. Finally, participants represented a variety of work-status segments including Stay-At-Home Moms (SAHM), Full-time (FT) employed and Part-time (PT) employed.

4.2.3. Mommy blog readers. The readers of the participating blogs were primarily comprised of women but one indicated having approximately $20 \%$ male readership. Their readers tended to be between the ages of 20 and 44 years old with most residing in the U.S. Little was known about the children of their readers. One participant shared that most of her readers' children were younger than 10 years old. The total number of readers (or followers) across all four blogs was approximately 50,000 .

\subsection{Procedures}

4.3.1. Pre-intervention. After receiving approval by the University Institutional Review Board, the blogs of the interested bloggers from TheMotherhood network were reviewed and vetted by the research team. Bloggers were deemed ineligible if they had posted content that suggested they may not be in support of vaccination and/or a health-related topic would not fit with the normal content of their blogs, e.g. some only posted about coupons and product giveaways. The remaining nine bloggers were contacted by email to request their participation. Five agreed to participate in the study and were asked to review the informed consent before completing the initial questionnaire. One participant requested to respond to the questionnaire over the phone; all others completed the questionnaire online via a Qualtrics link. Participants were given 2 weeks to respond to the initial questionnaire.

4.3.2. Intervention. At the conclusion of the questionnaire, participants were asked whether they would like to participate in the intervention component of the study. Out of five participants, four consented to continue. They were provided CDC-developed content including key messages about HPV and the vaccine and four visual stimuli. A draft blog post was also written and made available upon request. The post was drafted to be consistent with the CDC key messages, used gain-framed messages [47][15], and was written in plain language to discuss the vaccine in terms of mothers' real life experiences (e.g. worrying about children, desire to protect them and feeling overwhelmed by all the decisions parents need to make). The bloggers were sent occasional emails by the research team to ensure their continued interest and provide support or messaging guidance as needed. Participants were given 2 weeks to complete their post and make it live on their blog.

4.3.3. Post-intervention. Following completion of the posts, all four participants were asked to complete a post-study questionnaire. One participant also provided additional feedback via email to the research team. At the conclusion of the campaign, reader 
comments on each blog post were reviewed and estimated earned impressions were tabulated.

\subsection{Analysis}

In an effort to measure process outcomes, the researchers analyzed a variety of data sources. Frequency and thematic analyses were performed on the pre- and post-intervention questionnaire data to derive greater understanding of the mommy blogger demographics, their readership demographics and how the intervention impacted HPV vaccine knowledge or attitudes of the blogger. A proprietary calculation, which assesses content posted online, length of time live, and what assets, or content types, are posted online was used to estimate earned impressions for the campaign through December 14, 2015 [48].

\section{Results}

\subsection{Blogger perspectives}

5.1.1. Pre-intervention. Prior to starting the study, the researchers' analysis of the bloggers' blog content revealed there was no HPV vaccination information currently posted. Three out of four participants indicated that their readership would appreciate receiving HPV information from them. One participant indicated having some hesitancy, as "vaccination is a hot topic." The participants shared that they would be motivated to write about HPV because of the importance of preventing cervical cancer and the need to reach people before their children reach "critical milestones." However, this sentiment was balanced with the need to present the topic in a factual manner, not to stir up controversy, and to ensure that the content was in fact "good for [the blogger's] readers and their children."

When asked about specific messaging strategies, three out of four participants mentioned 'protection' or 'safety' as an important theme. At least two also mentioned the importance of informed decision making. One participant suggested including messages about:

"What [the vaccine] is, why kids need it, and a serious discussion on the risks. Perhaps a paragraph on making an informed decision yourself. I think knowledge is power - no matter what your final personal decision is."

All four participants utilizing the online survey indicated they would be willing to participate in the intervention, write a blog post and complete the postintervention follow up questionnaire

One participant requested to be interviewed by phone, in lieu of responding to the online questionnaire, and provided some additional feedback for this type of campaign. She shared a recent experience about being commissioned, by a national pharmacy, to write a post about flu season to promote the influenza vaccine. She indicated being frustrated with the "false-arguments" and "name calling" that ensued in the online conversations that followed her post:

"I really don't think we're changing people's minds that aren't willing to vaccinate, so it just depends on if I am in the mood to deal with the responses... They go to their natural news or their website and copy and paste a whole bunch of stuff into a comment. Then if I delete a comment I am called closed-minded."

This blogger indicated having concern not just for the reputation of her site but also a desire to "not perpetuate the lie." She stated that she was willing to tolerate the comments about the influenza vaccine because she was compensated for her post, but that she hesitated to agree to participate in this intervention. When asked how she might frame an HPV message if posting on her blog she stressed the importance of tailoring the message to her specific readership, e.g. parents with special needs children, and to provide a rationale for vaccination that is in line with their values and their children's health needs.

While specific knowledge measures were not utilized for this study, all four participants who agreed to post content requested the provision of information or resources to guide them - a common practice in the blogosphere [49]. One participant indicated she would write her own post but use the key messages and graphics provided by the research team. The other three participants utilized the draft blog post provided by the research team with some minor modifications discussed in a subsequent section.

5.1.2. Post-intervention. In the follow-up questionnaire, participants were asked about their perceptions having written a blog post and any reader commentary they received. Two participants indicated that sticking to the facts was highly important versus providing personal opinion, while another blogger felt that a narrative approach that her readers could relate to was more effective. One blogger also noted that a useful strategy in the future may be to write a post on how get the "conversation rolling with your tween."

Of the participants who received comments (2 of $4)$, they indicated being pleased with the reader 
responses. One participant also shared that in light of her post, she had a couple of offline conversations with friends and family. She mentioned that her 15-year-old son engaged in a conversation with her about the topic and joked that she should title her blog post the "stuff no one wants to talk about." She also noted that friends shared their own experiences with HPV vaccination - one related to a healthcare provider recommendation and another related to health insurance - both of which she called "very enlightening." More details on responses are provided in a subsequent section.

When asked about whether they would be willing to write a post about the HPV vaccine in the future, only three participants responded. One responded in the affirmative while the other two responded with "probably not" or "it would depend on how busy I am and on compensation."

\subsection{Blog post framing}

Following the content analysis and the initial questionnaire, it was shown that all four of the participants have at least some experience with writing about health topics and all four indicated that their readers appreciate health-related content. Although all participants thought HPV vaccination information would be of interest to their readers, none of them had written about the issue previously.

5.2.1. Blog post \#1. This post utilized the blog post draft developed by the research team including the title "Checking One Thing Off My Worry List: A Perspective on the HPV Vaccine". The blogger did not include any of the images provided by the research team, but rather used an image of a late adolescent female (or young adult) reclining on a couch and working on a computer. The content of the post was almost identical to the draft provided and received one comment. The blogger also shared her post on Facebook and Twitter.

5.2.2. Blog post \#2. This post also utilized the blog post draft developed by the research team with no substantive modifications to the content or title. The blogger included one of the images provided by the researchers, which was a CDC advertisement about cancer prevention with an adolescent girl pictured. She also included an image of her own, which appeared to be a pre-adolescent girl wearing jeans and a hoodie, contemplatively looking up. This blog post did not receive any comments.
5.2.3. Blog post \#3. This post utilized the blog post draft developed by the research team with no modification to the title and one revision to the content.

Original content: So why wouldn't you want to vaccinate against this preventable cancer if you could? I know I want to.

Revised to: Wouldn't you want to vaccinate against this preventable cancer if you could?

The blogger also chose to incorporate her own imagery, which appeared to be photographs of her digging in the garden with her young daughter and son and then another image of her three children walking hand in hand across a field.

5.2.4. Blog post \#4. This post utilized some of the key messages and images provided by the research team but were contextualized by the blogger's own content. She started the post by reminding her readers that she often shares information about parenting and healthy living but recognizes how difficult those decisions can be. She also discusses how the health of her children is a top priority. She points to CDC as a reliable and understandable source before providing some facts about HPV as a STI. The post includes facts about how common HPV is, how most people are asymptomatic and how the body typically fights off the infection. Then she points out the fact that HPV can "sometimes" lead to cancer in both men and women. Next she provides some statistics about disease incidence and provides links to the $\mathrm{CDC}$ vaccine information sheet.

She acknowledges that vaccination "can be a hotbutton item" but that she only wants to "share information so [parents] can make an informed decision that is right for you and your family." She explains the timing of the vaccine using language provided by the research team and supplements it with the following explanation:

"HPV vaccines offer the best protection to boys and girls who receive all three doses and have time to develop an immune response before they become sexually active. I have some friends that are afraid that by talking with their kids about this, it's like opening the door to sex."

She uses this statement to connect to additional CDC messaging provided by the research team about closing the door to cancer. Next she provides guidance on how to incorporate the vaccine into "the talk" (about sexual health and reproduction) and notes that it is not easy, stating "precious little about parenting is right?" Following publication of the post, she edited the conclusion to remind parents to check with their insurance company about coverage options. The 
blogger utilized two images from the research team (boy HPV ad and HPV infographic) and received two comments on her post. She also shared the post on Twitter.

\subsection{Campaign reach and reader response}

The campaign generated an estimated 3,601,790 earned impressions in total. The first blog posts went live on November 9th, 2015 and all of them remained online through December 14th, 2015 (the date of completion of data collection). Given the varying dates of publication, it accounts for an average of 33 days online per post. Content, images and hyperlinks were most commonly used and three out of the four posts were shared on either Facebook or Twitter.

No data were collected directly from the blogs' readership however the comments publicly posted were incorporated into the evaluation. Two blog posts received comments for a total of three comments. All comments posted were positively valenced, and in some cases provided personal anecdotes to support the points and rationale made in the post. In response to blog \#1, a reader shared a story about her daughter's difficult health experience and how she wished the vaccine had been available before:

"I WISH this had been around for my daughter, back when she was $17 \ldots$ During a routine exam, she had pre-cancerous cells on her cervix. After a year of exams, doctors, freezing off things, she was deemed cancer-free. Jump ahead another 6 years... after trying to have a baby for the first four years of marriage they are thankfully adopting. The scarring from her [cancer] kept her from having children. The vaccine would have been a God-send, and saved miscarriages, and heartache. I'm all for it."

In response to blog \#4, one reader expressed her regret for missing out on an opportunity to vaccinate her sons for HPV:

"Ok, I was literally JUST talking with my doctor yesterday about this. He was SUPER neutral about if my boys should get them or not. So we left without getting them, now I am regretting it."

Another reader responded "YES!! Thank you for this!!"

Despite the large readership of these blogs, no negative comments were posted. Additionally, while there is not sufficient evidence to suggest a relationship, during the time of our intervention study, mentions about HPV vaccination online did increase. See Figure 1.

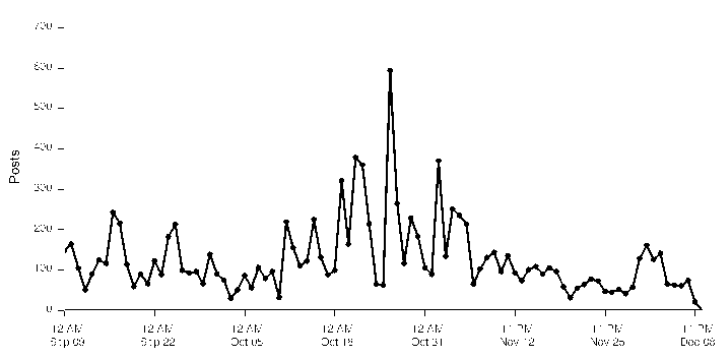

Figure 1. Online mentions about HPV vaccination between September and December 2015

\section{Lessons learned}

The preliminary results of this study and pilot campaign are promising for future vaccine promotion efforts. Mommy bloggers are uniquely positioned as trusted sources in their online communities and in many cases have broad reach (e.g. thousands of followers). Although vaccine knowledge and attitudes varied across the participants in this study, they were generally interested and willing to participate in an intervention study about a topic they felt was relevant to them and other mothers. The bloggers were easily contacted, timely in their responses and happy to utilize key messages provided as part of the campaign.

Much like their readers, each blogger has their own unique information needs and requires differing levels of support. Furthermore, their personal knowledge and attitudes about health topics, e.g. HPV vaccination, play a role in whether and how they are willing to communicate about it to others, e.g. some participants expressed hesitancy to write about vaccines because of potential backlash from their community. The reputations these mommy bloggers have built is critical to their livelihood and should be an important consideration of any campaign designer. Future campaigns should focus on providing mommy bloggers with a variety of messages and visuals to utilize while still allowing them flexibility in their approach.

Consistency of the campaign message is an important consideration that can be achieved by developing a strong relationship with the bloggers, keeping the lines of communication open and providing myriad messaging and visual options for them to use. Building a positive and communicative relationship with the bloggers is paramount to recruiting them to participate, ensuring accurate messaging, and addressing concerns as they arise.

Campaigns with short timeframes and/or bloggers with limited time may result in requests from bloggers to 'ghost write' posts that are only minimally revised. This was the case for three out of four of our participants. The other blogger (blog \#4) incorporated 
the CDC key messages and imagery while keeping the message true to her voice. She presented all the available facts and took the opportunity to communicate support to those with different perspectives and how it was important for each family to make their own decisions. Her personalized approach yielded the most follow up comments and an anecdote from one reader indicating her behavioral intentions changed as a result of the post. It was noteworthy that three of the four posts included imagery of young girls and only one chose to incorporate the boy CDC advertisement. This may suggest that the rhetoric of HPV as a woman's problem still exists and requires additional exploration.

\subsection{Limitations}

A significant constraint of this study was the time frame for study design, data collection, intervention and analysis. The sample size for the pilot study was also very small, which limited the diversity of the bloggers who participated and the feedback received from them. There was also no opportunity to develop, test and refine new messages throughout the course of this campaign. While three out of the four bloggers used the draft post provided, we were unable to systematically design or test the messages prior to dissemination. As well, the time frame for the study limited the analyses that could be conducted on mommy bloggers' attitudes and perceptions. Finally, due to the formative nature of this study and small sample, statistical significance could not be determined.

The study was designed for expediency and utilized two online questionnaires for collecting pre- and postintervention feedback from bloggers. Despite using open-ended questions in the questionnaires, participants provided concise responses with little detail. The opportunity to gain additional insight came through the ability to probe for more information during the pre-intervention interview conducted via phone and via email follow up with the bloggers. It is reasonable to assume that richer qualitative data on both the blogger attitudes and blog post decisions could have been achieved using an interview approach with all participants. It is important to note however that none of the participants received compensation or incentives for their participation in the study. Campaign designers of future similar intervention studies who wish to obtain richer data through the use of in-depth interviews should plan to compensate participants for their time.

\section{Conclusion and future research}

These preliminary results show promise for the utilization of mommy bloggers as opinion leaders in HPV promotion. While they represent another channel of public health communication, they also serve as an everyday opinion leader to thousands of mothers across the nation. Prior research has found that "the social network, including friends, family members, and media sources, are a key source of [health] information for many women" [50]. Findings from this pilot study support these findings suggesting that they are well positioned to deliver timely health information and work hand-in-hand with health campaigners to influence behavior. Correspondingly mommy bloggers also serve as the voice of their readership and have the ability to advocate for others. In this way, mommy blogger social networks can inform the decision making process for many women, specifically moms and parents.

Despite the small size of this pilot, participants shared that the experience extended beyond their interaction in the blogosphere and prompted conversations with their family and friends offline. If such a small pilot study with mommy bloggers can have an impact both within and beyond the blogosphere, then the investment in a larger study is worthy of serious consideration. As well, even though this campaign generated more than 3 million earned impressions, reach of the campaign would continue to increase with more time.

Future research should look to engage more bloggers over longer periods of time. Further research using a larger participant group, and/or a longer study timeframe would also address the statistical significance limitations of this study. As well, future work should include the creation and testing of new messages. As the network of mommy bloggers becomes increasingly diverse (including daddy bloggers), additional attention should be paid to the value of using tailored messages in more niche topical areas. To eliminate bias, reader comments from before the study should be compared to comments received during and after the intervention. Finally, both online and offline conversations that ensue as a result of a larger campaign should be tracked.

As it relates to the research questions posed for this study, the findings suggest that mommy bloggers' knowledge and attitudes about HPV vaccination were generally positively affected by the intervention (RQ1). Despite the fact that only one mommy blogger wanted to continue to write about the topic in the future, the reasons were not due to the intervention itself but fatigue from anti-vaccination reader comments. As well, the findings suggest that mommy bloggers frame HPV vaccine promotion messages to their readership 
based on facts and with a neutral tone (RQ2). Finally, through commenting and reader acknowledgement of offline conversations that were generated as a result of the intervention, the intervention resulted in increased knowledge and positive attitudes towards HPV vaccination (RQ3), although more work would be need to validate this finding. Ultimately, this highly approachable, dialogic forum for health information exchange may be just the ticket to shifting public health away from paternalistic messages toward patient-centered communication and engagement.

\section{References}

[1] Centers for Disease Control and Prevention, "Genital HPV infection fact sheet", Retrieved from http://www.cdc.gov/std/hpv/stdfact-hpv.htm, 2015a.

[2] National Cancer Institute, "Human Papillomavirus Vaccines", Retrieved from http://www.cancer.gov/about-cancer/causesprevention/risk/infectious-agents/hpv-vaccine-factsheet, 2015.

[3] E.F. Dunne, L.E. Markowitz, M. Saraiya, S. Stokley, A. Middleman, E.R. Unger, A. Williams and J. Iskander, "CDC grand rounds: Reducing the burden of HPVassociated cancer and disease", Mortality and Morbidity Weekly Report (MMWR), Retrieved from http://www.cdc.gov/mmwr/preview/mmwrhtml/mm630 4a1.htm, 2014, 63, pp. $69-72$.

[4] Centers for Disease Control and Prevention, "HPVAssociated cancers statistics", Retrieved from http://www.cdc.gov/cancer/hpv/statistics/, 2014.

[5] Merck, "Merck launches national advertising campaign for GARDASIL $®$, Merck's new cervical cancer vaccine" [News release], Retrieved from http://www.migrantclinician.org/toolsource/resource/me rck-launches-national-advertising-campaign-hpvvaccine.html, 2006.

[6] Centers for Disease Control and Prevention, "Recommendations on the Use of Quadrivalent Human Papillomavirus Vaccine in Males - Advisory Committee on Immunization Practices (ACIP)", Retrieved from http://www.cdc.gov/mmwr/preview/mmwrhtml/mm605 0a3.htm, 2011.

[7] E. Olshen, E.R. Woods, S.B. Austin, M. Luskin, and H. Bauchner, "Parental acceptance of the human papillomavirus vaccine", Journal of Adolescent Health, 2005, 37, pp. $248-251$.

[8] A.B. Alexander, N.W. Stupiansky, M.A. Ott, D. Herbenick, M. Reece, and G.D. Zimet, "What parents and their adolescent sons suggest for male HPV vaccine messaging”, Health Psychology, 2014, 33, pp. $448-$ 456.

[9] L. Brabin, S.A. Roberts, F. Farzaneh, and H. Kitchener, "Future acceptance of adolescent human papillomavirus vaccination: A survey of parental attitudes", Vaccine, 2006, 24, pp. 3097 - 3094.

[10] J. Waller, L.A.V. Marlow, and J. Wardle, "Mothers attitudes towards preventing cervical cancer through human papillomavirus vaccination: A qualitative study", Cancer Epidemiology, Biomarkers \& Prevention, 2006, 15 , pp. $1257-1261$.

[11] L.A. Marlow, J. Waller, and J. Wardle, "Parental attitudes to pre-pubertal HPV vaccination", Vaccine, 2007, 25, pp. 1945-1952.

[12] X. Nan, X. Zhao, and R. Briones, "Parental cancer beliefs and trust in health information from medical authorities as predictors of HPV vaccine acceptability", Journal of Health Communication, 2014, 19, pp. 100114.

[13] H.L. Gainforth, W. Cao, and A.E. Latimer-Cheung, "Message framing and parents' intentions to have their children vaccinated against HPV", Public Health Nursing, 2012, 29, pp. $542-552$.

[14] N.T. Brewer and K.I. Fazekas, "Predictors of HPV vaccine acceptability: A theory-informed, systematic review", Preventive Medicine, 2007, 45, pp. 107-114.

[15] A. Shafer, J.R. Cates, S.J. Diehl, and M. Hartmann, "Asking mom: Formative research for an HPV vaccine campaign targeting mothers of adolescent girls", Journal of Health Communication, 2011, 16, pp. 988 - 1005.

[16] R. Thomaselli, “As sales drop, fall brings big push for Gardasil vaccine: Merck ups marketing efforts as girls go for back-to-school checkups", Advertising Age, New York, NY, Retrieved from http://adage.com/article/news/merck-ups-gardasilvaccine-push-backschool-checkups/138364/, 2009.

[17] T.L. Thomas, and S. Snell, "Vaccinate boys with the HPV vaccine? Really?", Journal for Specialists in Pediatric Nursing, 2013, 18, pp. 165 - 169.

[18] S. Grantham, L. Ahern, and C. Connolly-Ahern, "Merck's one-less campaign: Using risk message frames to promote the use of Gardasil ${ }^{\circledR}$ in HPV prevention", Communication Research Reports, 2011, 28, pp. 318326.

[19] K.R. Ylitalo, H. Lee, and N.K. Mehta, "Health care provider recommendation, human papillomavirus vaccination, and race/ethnicity in the US national immunization survey", American Journal of Public Health, 2013, 103, pp. $164-169$.

[20] Centers for Disease Control and Prevention, "You are the key to cancer prevention", Retrieved from http://www.cdc.gov/hpv/hcp/, 2015c

[21] Centers for Disease Control and Prevention, "National immunization survey", Retrieved from http://www.cdc.gov/vaccines/imzmanagers/coverage/nis/child/infographic-2013.html, $2015 b$.

[22] S. Stokley, J. Jeyarajah, D. Yankey, M. Cano, J. Gee, J. Roark, C.R. Curtis, and L. Markowitz, "Human papillomavirus vaccination coverage among 
adolescents, 2007-2013, and postlicensure vaccine safety monitoring, 2006-2014 - United States", Mortality and Morbidity Weekly Report (MMWR), 2014,63 , pp. $620-624$.

[23] A.M. Griffioen, S. Glynn, T.K. Mullins, G.D. Zimet, S.L. Rosenthal, J.D. Fortenberry, and J. Kahn, "Perspectives on decision making about human papillomavirus vaccination among 11-to-12-year-old girls and their mothers", Clinical Pediatrics, 2012, 51, pp. $560-568$.

[24] J.M. Palefsky, "Human papillomavirus-related disease in men: Not just a women's issue", Journal of Adolescent Health, 2010, 46, pp. S12 - S19.

[25] D.L. Gustafson and C.F. Woodworth, "Methodological and ethical issues in research using social media: A metamethod of Human Papillomavirus vaccine studies", BMC Medical Research Methodology, 2014, 14, pp. 111.

[26] C. Zhang, M. Gotsis, and M. Jordan-Marsh, "Social media microblogs as an HPV vaccination forum", Human Vaccine and Immunotherapeutics, 2013, 9, pp. $2483-2489$.

[27] M. Dutta-Bergman, "Primary sources of health information: Comparisons in the domain of health attitudes, cognitions and behaviors", Journal of Health Communication, 2004, 16, pp. 273 - 288.

[28] J. Dworkin, S. Walker, J. Connell, and J. Doty, "Parenting 2.0 summary report: Parents' use of technology and the Internet", Retrieved from http://www.cehd.umn.edu/fsos/parent20, 2012.

[29] eMarketer," What Health Info Do Consumers Seek Online?", Retrieved from http://www.emarketer.com/Article/What-Health-InfoDo-Consumers-Seek-Online/1009698, 2013.

[30] M. Bailey, 'New survey reveals moms' media habits", Marketing to Moms Coalition and Current Lifestyle Marketing, 2008.

[31] Scarborough Research, Retrieved from http://mashable.com/2012/05/08/mommy-bloggerinfographic/, 2012.

[32] YourDictionary.com, Mommy blogger definition. Retrieved from http://www.yourdictionary.com/mommy-blogger, 2015.

[33] E. Chatman, "Diversity with Bloggers and Brands. Part I", CollectiveBias.com, Retrieved from http://collectivebias.com/blog/2015/01/diversitybloggers-brands-part-1/, 2015.

[34] eMarketer, Understanding How New Moms Share, Retrieved from http://www.emarketer.com/Article.aspx?R=1008056, 2010.

[35] J.M. Heisler, and J.B. Ellis, "Motherhood and the construction of "mommy identity": Messages about motherhood and face negotiation", Communication Quarterly, 2008, 56, pp. 445-467.
[36] W. Mitchell, and E. Green, “'I don't know what I'd do without our Mam' motherhood, identity and support networks", The Sociological Review, 2002, 50, pp. 122.

[37] J.A. Smith, "Identity development during the transition to motherhood: an interpretive phenomenological analysis", Journal of Reproductive and Infant Psychology, 1999, 17, pp. 281-299.

[38] A.M. Walsh, K. Hamilton, K.M. White, and M.K. Hyde, "Use of online health information to manage children's health care: A prospective study investigating parental decisions", Health Services Research, 2015, 15, pp. 793794.

[39] E.K. Brunson, "The impact of social networks on parents' vaccination decisions", Vaccine, 2013, 31, pp. $5466-5470$.

[40] J. Leask, S. Chapman, P. Hawe, and M. Burgess, "What maintains parental support for vaccination when challenged by anti-vaccination messages? A qualitative study", Vaccine, 2006, 24, pp. 7238-7245.

[41] S. Fox, "The social life of health information", Pew Research Center, Accessed at http://www.pewresearch.org/fact-tank/2014/01/15/thesocial-life-of-health-information/, 2014.

[42] L. Faw, "Is Blogging Really A Way For Women To Earn A Living?" Forbes.com, Accessed at http://www.forbes.com/sites/larissafaw/2012/04/25/isblogging-really-a-way-for-women-to-earn-a-living-2/, 2012.

[43] Punchbowl.com, "Why Mom Bloggers Are So Valuable to Brands", Accessed at http://www.punchbowl.com/trends/blog/post/why-mombloggers-are-so-valuable-to-brands, 2013.

[44] T.W. Valente, Social Networks and Health: Models, Methods, and Applications, Oxford University Press, New York, NY, 2010.

[45] G.L. Kreps, and L. Neuhauser, "New directions in eHealth communication: opportunities and challenges", Patient education and counseling, 2010, 78(3), pp. 329336.

[46] J.W. Creswell, Qualitative inquiry and research design: Choosing among five traditions (3rd ed.), Sage, Thousand Oaks, CA, 2011.

[47] M. Gerend and J. Shepherd, "Using message framing to promote acceptance of the human papillomavirus vaccine”, Health Psychology, 2007, 26, pp. 745-752.

[48] A. Burke, "Social media measurement for public health campaigns", PRNews Measurement Guidebook, PRNews, New York, NY, 2012, pp. 173-175.

[49] A. Burke-Garcia and G. Scally, "Trending now: future directions in digital media for the public health sector", Journal of Public Health, 2014, pp. 527-534.

[50] L. Yee, and M. Simon, "The role of the social network in contraceptive decision-making among young, African American and Latina women", Journal of Adolescent Health, 2010, 47(4), pp. 374-380 